\title{
Tuberculosis and HIV co-infection in children
}

\author{
Elisabetta Venturini ${ }^{1}$, Anna Turkova ${ }^{2}$, Elena Chiappini ${ }^{1}$, Luisa Galli ${ }^{1}$, Maurizio de Martino ${ }^{1}$, Claire Thorne ${ }^{3^{*}}$
}

\begin{abstract}
HIV is the top and tuberculosis is the second leading cause of death from infectious disease worldwide, with an estimated 8.7 million incident cases of tuberculosis and 2.5 million new HIV infections annually. The World Health Organization estimates that HIV prevalence among children with tuberculosis, in countries with moderate to high prevalence, ranges from 10 to $60 \%$. The mechanisms promoting susceptibility of people with HIV to tuberculosis disease are incompletely understood, being likely caused by multifactorial processes.

Paediatric tuberculosis and HIV have overlapping clinical manifestations, which could lead to missed or late diagnosis. Although every effort should be made to obtain a microbiologically-confirmed diagnosis in children with tuberculosis, in reality this may only be achieved in a minority, reflecting their paucibacillary nature and the difficulties in obtain samples. Rapid polymerase chain reaction tests, such as Xpert MTB/RIF assay, are increasingly used in children. The use of less or non invasive methods of sample collection, such as naso-pharyngeal aspirates and stool samples for a polymerase chain reaction-based diagnostic test tests and mycobacterial cultures is promising technique in HIV negative and HIV positive children. Anti-tuberculosis treatment should be started immediately at diagnosis with a four drug regimen, irrespective of the disease severity. Moreover, tuberculosis disease in an HIV infected child is considered to be a clinical indication for initiation of antiretroviral treatment. The World Health Organization recommends starting antiretroviral treatment in children as soon as anti-tuberculosis treatment is tolerated and within 2- 8 weeks after initiating it. The treatment of choice depends on the child's age and availability of age-appropriate formulations, and potential drug interactions and resistance. Treatment of multidrug resistant tuberculosis in HIV-infected children follows same principles as for HIV uninfected children. There are conflicting results on effectiveness of isoniazid preventive therapy in reducing incidence of tuberculosis disease in children with HIV.
\end{abstract}

Conclusion: Data on HIV/TB co-infection in children are still lacking. There are on-going large clinical trials on the prevention and treatment of TB/HIV infection in children that hopefully will help to guide an evidence-based clinical practice in both resource-rich and resource-limited settings.

\section{Global epidemiology of HIV and TB}

Worldwide, 2 billion people are estimated to be latently infected with tuberculosis (TB) [1], whilst there are an estimated 35.3 million people living with HIV, $70 \%$ of whom live in sub-Saharan Africa (SSA) [2]. HIV is the top and TB is the second leading cause of death from infectious disease worldwide [1]. Annually there are an estimated 8.7 million (range, 8.3-9.0 million) incident cases of TB and 2.5 million new HIV infections [1,2]. Of the 8.7 million incident cases of TB in 2011, 1.1 million (13\%) were among people living with HIV [2]. There are well established epidemiological and biological synergies

\footnotetext{
* Correspondence: claire.thorne@ucl.ac.uk

${ }^{3}$ Centre of Paediatric Epidemiology and Biostatistics, University College London Institute of Child Health, London, WC1N 1EH, United Kingdom Full list of author information is available at the end of the article
}

between HIV and TB, influencing the distribution, progression and outcomes of both infections. The HIV epidemic is a key factor behind the resurgence in TB incidence worldwide and HIV is the pre-eminent risk factor for the development of TB. One in eight incident cases of TB occur in HIV positive individuals, with a quarter of all TB deaths in people with HIV, while around a fifth of HIV related deaths occur in incident TB cases [2,3]. Much of the burden of TB and HIV codisease is in Africa, where one-third of the approximately 2.3 million people who developed TB in 2010 were HIV positive [1], although other regions strongly affected by TB and HIV dual epidemics are India and Eastern Europe [4,5].

The HIV epidemic has also had an impact on the age related prevalence of TB disease, with a lowering in the 
peak age groups affected, such that in high HIV prevalence areas, the peak TB incidence rates are now seen among adults aged 20-45 years [6].

\section{Children and HIV}

The impact of the HIV pandemic on children has been huge, and children account for around 10\% of new global HIV infections with an estimated 3.4 million children aged less than 15 years living with HIV in 2010 [7]. The survival of children in antiretroviral treatment (ART) era has dramatically improved in both resourcerich and resource-limited settings [8-12]. Although coverage with ART is improving, it is estimated that in low and middle income countries only around $34 \%$ of children aged $<15$ years needing ART are estimated to be receiving treatment, compared with $68 \%$ in adults $[2,7,13]$.

\section{Epidemiology of paediatric HIV/TB disease}

There are scarce data on the incidence of TB among HIV positive children and the prevalence of HIV among children diagnosed with $\mathrm{TB}$, and the information that is available is difficult to interpret due to problems with diagnosis, under-ascertainment and selection of study populations (i.e. most are recruited from hospitals or referral hospitals rather than the community). The World Health Organization (WHO) estimates that HIV prevalence among children with $\mathrm{TB}$, in countries with moderate to high prevalence, ranges from 10 to 60\% [7], with the prevalence varying depending on the background rates of HIV infection and estimates from several studies are summarised in Table 1 [14-21].

Estimated rates of TB among children with HIV also vary widely, partly depending on whether the study is taking place in a TB endemic area or not and on highly active anti-retroviral treatment (HAART) coverage in that area, but also due to the problems of reaching a definitive diagnosis of TB in children with HIV and under-ascertainment. In one large paediatric HIV clinic in London, UK, there was an average of two children with HIV per year who presented with active TB over a 15 year period $(18 / 328,5.5 \%$ of HIV infected patients were treated for active TB) [22] and in a US cohort of nearly $1500 \mathrm{HIV}$ infected children, TB disease was found $3 \%$, with an incidence of 0.61 per 100 child-years [23]. In contrast, in high prevalence countries, the incidence of TB disease in HIV positive children is much higher. Thus, in a South African retrospective study, incidence of TB disease was estimated to be 23 per 100 child-years among HIV positive children receiving HIV care [24]. With increasing coverage with ART, the incidence of TB has been decreasing, but remains substantially higher in HIV positive children than in the general paediatric population [25].

\section{Impact of HIV on pathogenesis of TB}

$\mathrm{HIV}$ alters the pathogenesis of $\mathrm{TB}$, increasing the risks of developing active TB in those with latent infection as well as in those newly exposed to TB. In the HIV-uninfected population, only around $10 \%$ of people infected with TB will develop TB disease [1]. However, in HIV positive people, there is a $20-30$ fold increased relative risk of developing TB disease from latent state compared with that in people without HIV [3], an increase that outweighs those of other risk factors such as malnutrition. The mechanisms promoting susceptibility of people with HIV to TB disease are incompletely understood. Risk of active TB increases with depletion of CD4

Table 1 Prevalence of HIV among children with TB [14-21]

\begin{tabular}{|c|c|c|c|c|}
\hline Study setting & Year & Study population & HIV prevalence & Reference \\
\hline Zambia & 1994 & Children with clinical TB diagnosis $(n=120)$ & $56 \%$ & Luo et al Tuberc Lung Dis 1994 [14] \\
\hline $\begin{array}{l}\text { Johannesburg, } \\
\text { South Africa }\end{array}$ & 2008 & $\begin{array}{l}\text { Cultured confirmed cases of TB in children } \\
\text { aged }<14 \text { years }(n=1317)\end{array}$ & $\begin{array}{l}52 \% \text { in children with drug- } \\
\text { susceptible TB; } 53 \% \text { in the } 13 \text { with } \\
\text { MDR-TB }\end{array}$ & Fairlie et al BMC Inf Dis 2011[15] \\
\hline Mumbai, India & 2002 & Children with disseminated TB $(n=68)$ & $16 \%$ & $\begin{array}{l}\text { Karande et al J Trop Pediatr } 2002 \\
\text { [16] }\end{array}$ \\
\hline $\begin{array}{l}\text { Rio de Janeiro, } \\
\text { Brazil }\end{array}$ & $\begin{array}{l}1999- \\
2008\end{array}$ & $\begin{array}{l}\text { Children attending a reference hospital } \\
(n=473)\end{array}$ & $17 \%$ (but only $56 \%$ tested for HIV) & Matos et al J Pediatr 2012 [17] \\
\hline $\begin{array}{l}\text { Cape Town, South } \\
\text { Africa }\end{array}$ & $\begin{array}{l}1999- \\
2004\end{array}$ & $\begin{array}{l}\text { Children born in } 1999 \text { and diagnosed with } \\
\text { TB in public health facilities up to } 2004 \\
(n=1607)\end{array}$ & $\begin{array}{l}37 \% \text { (but only } 16 \% \text { had HIV test } \\
\text { results available) }\end{array}$ & $\begin{array}{l}\text { Moyo et al Int J Tuberc Lung Dis } \\
2010 \text { [18] }\end{array}$ \\
\hline $\begin{array}{l}\text { Santo Domingo, } \\
\text { Dominican Republic }\end{array}$ & 1996 & $\begin{array}{l}\text { Children aged 18-59 months with clinical TB } \\
\text { diagnosis }(n=189)\end{array}$ & $5.8 \%$ & $\begin{array}{l}\text { Espinal et al J Acquir Immune Defic } \\
\text { Syndr Hum Retrovirol } 1996 \text { [19] }\end{array}$ \\
\hline $\begin{array}{l}\text { Durban, South } \\
\text { Africa }\end{array}$ & $\begin{array}{l}1998- \\
1999\end{array}$ & $\begin{array}{l}\text { Hospitalised children with culture-confirmed } \\
\text { TB aged }<13 \text { years }(n=118)\end{array}$ & $48 \%$ & $\begin{array}{l}\text { Jeena et al Int J Tuberc Lung Dis } \\
2002 \text { [20] }\end{array}$ \\
\hline $\begin{array}{l}\text { Abidjan, Côte } \\
\text { d'Ivoire }\end{array}$ & $\begin{array}{l}1994- \\
1995\end{array}$ & $\begin{array}{l}\text { Children aged 0-9 years with newly } \\
\text { diagnosed TB (out- and in-patients) }(n=161)\end{array}$ & $19 \%$ & Mukadi et al AIDS 1997 [21] \\
\hline
\end{tabular}


T-cells, but studies have also shown that HIV positive individuals in high $\mathrm{TB}$ incidence regions have an increased risk of developing active $\mathrm{TB}$ in the first year after HIV seroconversion, i.e. with high CD4 T-cell counts [26]. In addition, HIV positive individuals on ART with high CD4 T-cell counts continue to have an increased risk of developing TB compared with uninfected controls. This suggests that although the loss of cell-mediated immunity with HIV disease progression is likely to be an important factor with respect to the increased risk of active TB associated with HIV, this is likely to be caused by multifactorial processes.

Studies have demonstrated depletion of TB-specific CD4 $\mathrm{T}$ cells in peripheral blood and in the lung at early stages of HIV disease, suggesting that TB-specific adaptive immunity may be especially susceptible to HIVassociated immune damage [27]. The essential role of CD4 T cells in generation of granulomas, and the depletion of such cells with HIV disease progression, may explain the increased risk of extra-pulmonary TB (EPTB) in HIV-positive patients [28]. Furthermore, the failure of the declining CD4 $\mathrm{T}$ cell population to regulate and maintain granulomas is one of the mechanisms proposed to be behind the increasing risk of re-activation disease in latently infected individuals with HIV. Turning to innate immunity, apoptosis (programmed cell death) of an infected macrophage is an important host immune response to TB infection. Recent work has suggested that in the context of HIV infection, apoptosis of alveolar macrophages is decreased, possibly due to raised IL-10 (an anti-inflammatory cytokine) in the lung [29], which may be a mechanism behind the increased susceptibility of those with HIV to TB. Genetic variants are also known to influence TB development in HIV positive patients. A recent case-control study in Brazil showed the novel association between certain inflammasome gene polymorphisms (i.e. CARD8 genetic variants) and the development of TB infection in HIV positive subjects [30].

\section{Challenges of diagnosis of TB in children with HIV}

WHO guidelines state that diagnosis of TB in an HIV positive child should follow the same approach as for HIV-uninfected children, with taking into account the history of TB contact, clinical features suggestive of TB (such as prolonged cough, poor weight gain or weight loss), positive tuberculin skin test (TST) $\geq 5 \mathrm{~mm}$ considered to be positive in HIV infected individuals, and suggestive chest $\mathrm{X}$-ray signs [31].

Paediatric TB and HIV have overlapping clinical manifestations, including fever, weight loss and lymphadenopathy, which combined with persistent cough, could lead to missed or late diagnosis, or alternatively a misdiagnosis of either infection or concomitant infections with similar clinical features. TB generally has a more severe clinical presentation in HIV positive individuals than in those uninfected. The risk of EPTB or disseminated TB is increased in HIV positive adults, particularly with low CD4 $\mathrm{T}$ cell counts [28]. In a US study of HIV positive adults with EPTB, there was a significantly increased risk of central nervous system, meningeal or disseminated TB disease compared to lymph node disease in those with severe immunosuppression (CD4 $<100$ cells $/ \mathrm{mm}^{3}$ ) [32]. In the limited paediatric literature, studies have reported no significant differences in the frequency of EPTB between HIV positive and negative children $[20,21,33]$. However, others have suggested that HIV infected children with more advanced HIV disease are at higher risk of EPTB and combination of extrapulmonary and pulmonary TB disease $[34,35]$. Overall, the clinical presentation of TB in HIV infected children, may depend on degree of immunocompromise, with severe, disseminated forms more frequently found in patients with advanced HIV infection; however this link is less well reported than in adults.

The presence of HIV co-infection compounds the well-recognised challenges of reaching a definitive diagnosis in children with suspected TB. Although every effort should be made to obtain a microbiologically-confirmed TB diagnosis in children, in reality this may only be achieved in a minority, reflecting the paucibacillary nature of much childhood $\mathrm{TB}$, the inability of young children to expectorate sputum, the difficulties of obtaining gastric aspirates and the low yield of such samples. Sputum induction with hypertonic saline was found to be safe and useful for microbiological confirmation of pulmonary TB in both HIV positive and HIV negative children in a study in South Africa: overall, $24 \%$ of children had a positive smear or culture for Mycobacterium tuberculosis and induced sputum (IS) samples had a three-times greater yield than gastric lavage (GA), with no difference in yield by HIV infection status [36]. This was later evaluated in the community setting with $16.9 \%$ of clinically diagnosed TB cases confirmed microbiologically [37], highlighting the usefulness of this diagnostic tool in the outpatient settings. However the need for electrical supply and special technical equipment, together with the high work load and infectious hazard are substantial obstacles for widespread use of this method. It is important to note that low rates of microbiological confirmation in paediatric $\mathrm{TB}$ are in part due to low rates of obtaining the samples from children. For example, European Centre for Disease Prevention and Control (ECDC) surveillance demonstrated that only $42 \%$ of children with TB reported to ECDC had mycobacterial cultures sent in 2000-2009, however of those who had their cultures sent, $40 \%$ were culture positive [38]. It is important to highlight that every effort needs to be undertaken to collect samples from 
children with suspected TB for microbiological confirmation.

Rapid polymerase chain reaction (PCR) tests, such as Xpert MTB/RIF assay, are increasingly used in children with TB and are recommended by WHO as the initial diagnostic test in patients suspected of MDR-TB or HIV/TB [39]. The test is an important advance in rapid detection of TB disease and detection of drug resistance. The few prospective studies evaluating it in children have showed that it is much more sensitive than microscopy, with sensitivity being reported from 75 to $90 \%$ on sputum samples and nearly $70 \%$ on gastric aspirates, with comparable performance in HIV positive and HIV negative children [40,41]. Although the sensitivity of Xpert MTB/RIF test is higher than microscopy, a substantial proportion of children with negative test had positive MTB cultures. Hence Xpert MTB/RIF test cannot be used to rule out TB, and MTB culture remains a necessary diagnostic tool.

The use of less or non invasive methods of sample collection, such as naso-pharyngeal aspirates (NPA) and stool samples for a PCR-based diagnostic test tests and MTB cultures is promising technique which was evaluated in HIV negative and HIV positive children. Small pilot studies in South Africa reported on Xpert MTB/ RIF assay on stool in childhood TB. The first study assessed Xpert on decontaminated stool sediment. It showed that stool Xpert was equally sensitive to the assay on GA, detecting $75 \%$ of children with intra-thoracic culture confirmed TB [42]. In another pilot study, stool Xpert, which was performed directly on stool detected $47 \%$ culture-confirmed TB (80\% in HIVinfected children and 33\% in HIV-uninfected children) compared to $65 \%$ cases detected by Xpert on IS [43]. The study used small stool volumes, and the authors concluded that the sensitivity may be increased by an improved protocol. Another paediatric study from the South Africa compared NPA with IS: the sensitivity of two Xpert MTB/RIF tests were comparable (71\% vs $65 \%, \mathrm{P}=0.444)$, however the culture yield from NPA was significantly less than from IS $(96.6 \%$ vs $70.1 \%, \mathrm{P}<$ 0.001) [44]. Stool and NPA can be useful add on or alternative specimens in the settings where is unavailable and in older children in whom GA are difficult to obtain. Urine lipoarabinomannan has been recently shown to be useful in diagnosing TB in severely immunocompromised adults, detecting up to $61 \%$ of cultureconfirmed TB cases with CD $4<50$ cells $/ \mathrm{mm}^{3}$ [45]. There are no reported studies in children to date.

With respect to TST, there is a high rate of false negative results in HIV positive children. In a study in Cape Town, among nearly 300 paediatric TB cases, those with HIV were significantly less likely to have a positive TST than those HIV negative (36\% versus 59\%)
[18]. In a further study in South Africa among HIVinfected children with culture-confirmed TB, only 56\% had a positive TST [35]. There is considerable discrepancy between current recommendations on use of interferon- $\gamma$ release assays (IGRA) and TST in diagnosis of TB infection and TB disease in children [46]. A systemic review and meta-analysis on IGRA in childhood tuberculosis showed improved specificity of IGRA compared with TST and similar accuracy between IGRA and TST in detection of TB infection or TB disease; however the tests showed high level of discordant results and had lower sensitivity for HIV positive children [47]. In a recent South African study comparing performance of the TST and IGRA in children recruited from hospital outpatient settings, while the proportion with a positive TST did not vary according to HIV infection status, HIV positive children were significantly less likely to have a positive IGRA than HIV negative children after adjusting for degree of TB exposure; in addition, HIV negative children had an increasing probability of a positive TST with increasing age compared to HIV positive children, after adjusting for degree of TB exposure, Bacillus Calmette-Guérin (BCG) vaccination, malnutrition and past TB [48]. The authors concluded that caution is needed in interpreting IGRA results in the context of HIV infection, given their findings that the test performance compared with TST is differentially affected by age and HIV infection. Some studies demonstrated that enzyme-linked immunospot assay is more sensitive than TST in detection of active TB in HIV positive children however the sensitivity was not sufficiently high to rule out active TB $[49,50]$.

Although in adults with TB and HIV, particularly those with severe immunodeficiency, there may be atypical chest X-ray findings, the limited studies in children have not identified key differences in the radiological presentation of TB according to HIV status $[20,51,52]$. HIV positive children may frequently have other respiratory opportunistic infections such as Pneumocystis jirovecii pneumonia, lymphoid interstitial pneumonitis or other bacterial or viral pneumonias, which can further complicate diagnosis of pulmonary $\mathrm{TB}$, for example, due to similar clinical or radiological manifestations. However, the presence of one such opportunistic infection does not preclude concurrent infection with another, and the challenge is to identify all respiratory infections present and to treat appropriately.

In a case series of 18 children with HIV and active TB in London, nearly half of the children had not yet been diagnosed with HIV at the time they presented with TB [22]. The new directions in diagnosis of paediatric TB and novel TB diagnostic tests which are under development have been recently reviewed by Whittaker and colleagues [53]. 


\section{Clinical management of HIV/TB in children Prognosis}

Many paediatric studies reported that children experiencing HIV/TB co-disease have more severe disease and higher mortality than HIV negative children, reflecting the dual impact of the two infections. In the pre-ART era, very high mortality rates were reported for children with TB and HIV: in a retrospective study of HIVinfected children with culture confirmed TB in South Africa, $21 \%$ of children died whilst on TB therapy and a further $18 \%$ after TB treatment was competed, mostly (in $82 \%$ cases) due to causes other than TB [35]. In a cohort study in Ethiopia, children with HIV infection were around six times more likely to die from TB than those without HIV [52]. In a study in Côte d'Ivoire, the mortality rate in HIV-infected children with TB was $23 \%$ versus $4 \%$ in HIV-uninfected children, a 3.6 times increased risk; of note all deaths among the children with HIV occurred in those with severe immune suppression [21]. Not all paediatric studies showed higher TB associated mortality in HIV co-infected children: in a Zambian autopsy study of 180 HIV positive and 84 HIV negative children who died of respiratory disease, $26 \%$ of the HIV-negative and $18 \%$ of the HIV positive group died with TB (no significant difference), half of the HIV positive children had more than one respiratory disease or infection identified [54].

ART has modified HIV/TB co-infection epidemic and had a great impact on TB disease incidence, morbidity and mortality. In a study in the Democratic Republic of Congo, ART halved the hazard of developing TB in HIV-infected children [55], with a TB incidence rate in those receiving ART of 10.2 per 100 person-years versus 20.4 per 100 person-years in those not yet on ART; after 12 months of ART TB incidence decreased to 5.3 per 100 person-years. A recent prospective cohort study conducted in Kenya on 689 HIV-infected children aged 6 weeks to 14 years, showed that longer time on ART and longer duration of being in care before ART were associated with lower prevalence of $\mathrm{TB}$ at enrolment (adjusted hazard ratio [aHR] 0.91, P $=0.003$ and aHR $0.87, \mathrm{P}=0.001$ respectively) and lower incidence of $\mathrm{TB}$ during the study (aOR 0.91, $\mathrm{P}<0.001$ and aOR $0.92, \mathrm{P}<$ 0.001 respectively) [56]. These findings are consistent with those from a South African retrospective cohort study that reported that among children receiving ART, incidence of clinically diagnosed TB declined from 21.1 per 100 person-years during their pre-ART follow-up to 6.4 per 100 person-years after ART initiation [57]. A recent retrospective study from Soweto, South Africa, showed that scaling-up of the public-funded antiretroviral treatment program resulted in $70.6 \%$ reduction of culture-confirmed TB in HIV infected children in 4-year period from 2005 to 2009. However in 2009 the incidence of culture confirmed TB, was still 42-fold times higher in HIV positive compared to HIV negative children $(460.7 / 100,000$ vs. $11.0 / 100,000)$. Over three quarters of co-infected children were severely immunocompromised [58]. Over $40 \%$ decline of TB incidence was also observed in HIV negative children which maybe in part due to increased ART coverage in adults and the associated decreased TB transmission.

\section{Treatment}

Anti-TB treatment should be started immediately at TB diagnosis. In the most recent guidance, WHO recommends that all HIV positive children are started on four-drug TB treatment, irrespective of the TB disease severity (isoniazid, rifampicin, pyrazinamide and ethambutol) and that intermittent therapy should be avoided $[13,59]$.

TB disease in a HIV infected child is considered to be a clinical indication for initiation of ART [59]. WHO 2013 recommends starting ART in children as soon as TB treatment is tolerated and within 2- 8 weeks after initiating it [59]. The early start of ART is especially relevant for children with moderate-to-severe immunocompromise. The recommendations are extrapolated from adult trials, which showed that mortality is decreased in severely immunocompromised patients with CD $4<50$ cells $/ \mathrm{mm}^{3}$ with early start of ART [60-62]. A paediatric retrospective study from Johannesburg, South Africa, in children, most of whom were severely immunocompromised, showed that delaying ART for longer than 8 weeks was associated with increased mortality and worse virologic outcome [63]. The data from adult studies suggest that delayed ART in patients with no or mild immunocompromise was not associated with worse outcomes [60-62]. Therefore some clinicians consider delaying ART in children with no evidence of immunocompromise until TB treatment is well tolerated or even until the end of TB treatment in order to avoid potential drug interactions and significantly increased pill burden [22].

The choice of ART depends on the child's age and availability of age appropriate formulations, whether the child is already receiving treatment, their previous ART exposure, and potential drug interactions. Significant drug interactions occur with rifampicin, which is a potent CYP3A4 inducer, with a co-administration with nevirapine and protease inhibitors which are metabolised through cytochrome C450 enzymes. This may lead to a marked decrease in plasma concentration of these drugs; therefore in order to maintain therapeutic levels dose adjustment may need to be done when starting and stopping rifampicin. The regimen of choice in 
children aged over 3 years is efavirenz (EFV)-based $\mathrm{ART}$, as EFV has less significant drug interaction with rifampicin and dose adjustment is not required. EFV capsule sprinkles have been recently licensed in children aged over 3 months based on results of a paediatric population model [64]. However EFV capsules are not widely available in high HIV and TB prevalence settings, and further studies are required to assure the efficacy of EFV-based combinations in HIV/TB co-infected young children.

If lopinavir/ritonavir based ART is used, to overcome drug interactions with rifampicin, it will be necessary to increase ritonavir to $1: 1$ of lopinavir/ritonavir $[65,66]$. In settings where therapeutic drug level monitoring is available, dose adjustment of rifampicin and lopinavir or other protease inhibitors can be made based on the results. If available, rifabutin, a less potent CYP3A4 inducer, should be used instead of rifampicin to reduce drug interactions. ART-naïve children under 3 years of age can be started on nevirapine-based ART but without lead-in dose and maximum recommended dose (200mg/ $\mathrm{mm}^{3}$ twice daily) [59]. Some clinicians choose to increase the maintenance dose of nevirapine by $20-30 \%$ in order to reach an optimal exposure [67].

An alternative regimen in children is triple nucleoside reverse transcriptase inhibitors administered for the duration of anti-TB treatment [59]. This regimen has less virological efficacy but no significant drug interaction with rifampicin. It has been shown in a recent paediatric trial in Uganda and Zimbabwe to be immunologically and clinically similar to non-nucleoside reverse transcriptase inhibitors based ART and therefore may be valuable in children with controlled HIV infection who develop TB [68]. Information on drug interactions and advice on dose modification can be found at www.hiv-druginteractions.org.

There remains some debate with respect to duration of TB therapy in HIV-infected children. There is no evidence that HIV-infected children should have longer duration of treatment than HIV uninfected children if a good response to treatment is achieved. Generally HIV infected children without severe TB disease can receive standard duration of anti-TB treatment for 6 months, and the duration of anti-TB treatment can be extended in cases with suboptimal response to treatment [31]. However in the latter cases, poor adherence, suboptimal drug levels secondary to insufficient dosing or drug interactions, drug resistance and immune reconstitution inflammatory response should be considered and addressed appropriately.

\section{Multi drug resistant (MDR)-TB}

There are limited data on the extent of MDR-TB among HIV positive children. In one study in the Western Cape Province, South Africa, 5\% of HIV-infected children aged
$<14$ years with culture confirmed TB had MDR-TB [35]. A cross sectional study in children with culture confirmed TB in a setting with high prevalence of HIV (Johannesburg, South Africa) showed no difference in HIV prevalence in TB compared to MDR-TB (52.1\% vs. $53.9 \%$ respectively), suggesting there is no association between HIV infection and MDR-TB [15]. MDR-TB can be devastating in HIV infected individuals as demonstrated by the very high mortality rates in HIV positive adults in early institution-based outbreaks with MDR-TB and extensively drug resistant (XDR)-TB [69]. Advances in treatment of both HIV and drug resistant TB, combined with comprehensive care, have facilitated very good treatment outcomes in HIV infected children with MDR-TB and even XDR TB [70,71]. A retrospective study from Western Cape Province, South Africa, on children with culture-confirmed MDR-TB, with HIV prevalence of $43 \%$, overall treatment success was achieved in $82 \%$; multivariable analysis showed no significant association of HIV infection with poor treatment outcome (OR 1.46 [95\% CI, 0.46-4.63]; $\mathrm{P}=0.52$ ) [72].

Treatment of MDR-TB in HIV infected children follows the same principles as for HIV uninfected children as well outlined in the recent guidelines [73-75]. Based on extrapolations from adult studies, children with MDR-TB are recommended to receive TB treatment for 18-24 months regardless of their HIV status [75]. However, good results have been demonstrated with shorter and less intensive regimens in children with non-severe forms of TB disease [76]. Children with HIV/TB coinfection should be monitored closely for potential overlapping and added toxicities of ART and second line TB drugs. Attention to nutrition and adherence support with provision of directly observed therapy are extremely important.

\section{Immune reconstitution inflammatory syndrome (IRIS)}

Following initiation of ART in HIV/TB co-infected patients, IRIS may develop - presenting with either clinical manifestation of previously undiagnosed TB or paradoxical exacerbation of already diagnosed TB disease in spite of already initiated anti-TB treatment [77]. It usually occurs in severely immunocompromised patients and presents within 3 months of starting ART. It is frequently associated with a substantial fall of viral load and increase in CD4 count. Non-inflammatory drugs in mild cases or steroids in moderate-to-severe cases are usually required in the addition to perseverance with TB and HIV treatment. Most patients can be managed without stopping ART.

\section{Perinatal TB/HIV}

African studies carried out in the pre-ART era demonstrated that recent pregnancy was associated with 
increased risk of developing active TB in HIV positive women, which is associated with increased risk of maternal mortality [78]. Infants of mothers with active $\mathrm{TB}$ are at high risk of acquiring TB infection (usually postnatally) and developing active disease [79]. The extrapulmonary forms of the disease, miliary and meningeal TB, are greater risk factors for congenital TB [80]. In one study, at least $15 \%$ of mothers with active $\mathrm{TB}$ in pregnancy had transmitted infection to their infants by age three weeks [81]. In an Indian study of HIV-infected postnatal women not receiving isoniazid $\mathrm{TB}$ preventative therapy (IPT), there was a high maternal active TB incidence ( 5 cases/100 person-years), and infants of mothers with incident TB had a 3 -fold increased mortality rate compared with other infants [82]. Transmission and outcomes are affected by duration of therapy before delivery. Four months or more of therapy is protective to the foetus. However, noncompliance with therapy carries an increased risk of transmission of the Mycobacterium tuberculosis to the infant [83]. Further research to guide policies for TB screening and IPT in pregnant women with HIV is needed.

\section{Prevention}

\section{Isoniazid preventive therapy}

There are controversial results on effectiveness of TB preventive therapy in reducing incidence of TB disease in children with HIV. Zar and colleagues in a South African clinical trial including nearly 300 children showed that IPT halved mortality ( $8 \%$ versus $16 \%$ ) and reduced TB incidence from $10 \%$ to $4 \%$, reducing the chance of developing TB (confirmed or probable) by $72 \%$ [84]. However a recently conducted large double-blind, randomized, placebo-controlled trial of pre-exposure isoniazid prophylaxis against tuberculosis on 548 HIV infected children and 804 HIV uninfected children immunized with $B C G$ vaccine, showed no significant difference in the combined incidence of tuberculosis infection, tuberculosis disease, or death between the isoniazid group and the placebo group irrespective of HIV status [85].

The differences in coverage of ART and lack of exposure to TB disease are the likely explanations for the contrasting results, which was further underlined by Mark Cotton of Stellenbosch University who participated as an investigator in both trials [84-86].

Recently, results on the use of 3 months of rifapentine and isoniazid once weekly for people living with HIV showing higher treatment completion rates and better tolerance than 9 months of isoniazid administered daily. This regimen was as well tolerated in HIV-infected as in HIV negative individuals. Although increased risk of selection for rifampicin resistance was observed in the rifapentine/isoniazid group, the numbers were too few for conclusions to be drawn [87].

\section{BCG and the HIV-infected Infant}

While the risks of adverse events associated with BCG vaccination in HIV negative infants are low $(<0.04 \%$ for local disease and $0.0002 \%$ for disseminated disease), HIV positive infants have a markedly increased risk of developing local (5.6\%) and disseminated BCG disease (dBCG) (0.2\%) [87]. Recently the WHO assessed the risk of $\mathrm{dBCG}$ disease in HIV-positive infants to be approaching 1\% [31]. Moreover HIV infection severely impairs the BCG-specific $\mathrm{T}$ cell responses during the first year of life, giving little protection against tuberculosis in HIV-infected infants. Considering the significant risk of dBCG disease, these data strongly support the WHO recommendation of not giving BCG to children who are known to be infected with HIV [31]. However, in settings where limited resources are available, BCG vaccination is given at birth to all infants regardless of HIV exposure, considering the high endemicity of tuberculosis in populations with high HIV prevalence. Close follow up of infants known to be born to HIV-infected mothers and who received BCG at birth is recommended in order to provide early identification and treatment of any BCG-related complication [31].

\section{Conclusion}

TB and HIV both represent major threats to public health worldwide. Renewed global interest led to advances in recognising TB/HIV co-infection and understanding the mechanisms promoting susceptibility of HIV-infected people in developing TB disease as part of a multifactorial process [26-30]. Diagnosis of HIV/TB co-infection in children is still challenging. Paediatric TB and HIV have overlapping clinical manifestations, which could lead to missed or late diagnosis. TB disease in a child should alert the clinician to the possibility of HIV infection, particularly in high HIV prevalence settings, where HIV counselling and testing to the child and family should be strongly recommended. Difficulties in TB diagnosis in children are mostly related to their paucibacillary nature. New tests based on PCR have been developed, being applied in samples that require less invasive procedures such as stool and NPA and showing to be useful especially in settings in whom GA are difficult to obtain. Larger studies are needed to establish the sensitivity of these tests to rule out TB in children [42-44]. Few data are available about IGRA and TST in HIV positive children, showing high level of discordant results and lower sensitivity in these patients. Further well-designed and comparative studies on use of IGRA in immunocompromised children are required [46-50].

Following WHO 2013 recommendations, anti-TB treatment should be started immediately after diagnosis whereas ART could be delayed to 2-8 weeks after or as 
soon as TB therapy is tolerated. The regimen of choice should be carefully addressed considering age, drug interactions, especially if rifampicin is used, or MDR-TB cases [13,59].

There are controversial results on effectiveness of preventive therapy in reducing incidence of TB disease in HIV children. The last CROI meeting in 2013 reported that primary isoniazid prophylaxis seemed ineffective in preventing $\mathrm{TB}$ infection in young HIV-perinatally exposed infants $(<12$ months) in TB endemic regions [86]; nevertheless treatment of latent TB with isoniazid was effective in avoid developing TB disease in HIV infected children over 24 months of age [86]. Thus the questions remain regarding the protective effect of IPT in low TB burden settings. Moreover, shorter regimens have been studied in adults, i.e. 3 months rifapentine and isoniazid once weekly, with promising preliminary results on efficacy and compliance [87]. There are ongoing large clinical trials (i.e. the PREVENT TB study, SHINE-trial) on the prevention and treatment of TB/ HIV infection in children that should provide new, much needed data on paediatric TB/HIV infection and help to guide evidence-based clinical practice in both resource-rich and resource-limited settings.

\section{List of abbreviation used}

ART: anti-retroviral treatment; BCG: bacillus CalmetteGuérin; dBCG: disseminated BCG disease; ECDC: European Centre for Disease Prevention and Control; EFV: efavirenz; EPTB: extra-pulmonary TB; GA: gastric aspirate; IGRA: interferon $-\gamma$ release assays; IPT: Isoniazide preventing therapy; IRIS: Immune reconstitution inflammatory syndrome; IS: induced sputum; MDR: multi drug resistant; NPA: naso-pharyngeal aspirate; PCR: polymerase chain reaction; SSA: sub-Saharan Africa; TB: tuberculosis; TST: tuberculin skin test; WHO: World Health Organization; XDR: extensively drug resistant

\section{Competing interests}

The authors declare that they have no competing interests

\section{Declarations}

Funding for this supplement came from the Italian Health Ministry /Young Research Project.

This article has been published as part of BMC Infectious Diseases Volume 14 Supplement 1, 2014: Highlights in Pediatric Tuberculosis. The full contents of the supplement are available online at http://www.biomedcentral.com/ bmcinfectdis/supplements/14/S1

\footnotetext{
Authors' details

'Department of Health Sciences, Meyer Children University Hospital, University of Florence, Florence, Italy. ${ }^{2}$ Department of Paediatric Infectious Diseases, St Mary's Hospital, Imperial College NHS Trust, London, United Kingdom. ${ }^{3}$ Centre of Paediatric Epidemiology and Biostatistics, University College London Institute of Child Health, London, WC1N 1EH, United Kingdom.
}

Published: 8 January 2014
References

1. World Health Organization: Global tuberculosis report 2012.[http://apps. who.int/iris/bitstream/10665/75938/1/9789241564502_eng.pdf].

2. Global report: UNAIDS report on the global AIDS epidemic 2013. [http:// www.unaids.org/en/media/unaids/contentassets/documents/epidemiology/ 2013/gr2013/UNAIDS_Global_Report_2013_en.pdf]

3. Time to act. Save a million lives by 2015. Prevent and treat tuberculosis among people living with HIV. [http://www.stoptb.org/assets/documents/ resources/publications/acsm/TB_HIV_Brochure_Singles.pdf].

4. Swaminathan S, Ramachandran R, Baskaran G, Paramasivan CN, Ramanathan U, Venkatesan P, Prabhakar R, Datta M: Risk of development of tuberculosis in HIV-infected patients. Int J Tuberc Lung Dis 2000, 4:839-844.

5. Kruk A, Bannister W, Podlekareva DN, Chentsova NP, Rakhmanova AG, Horban A, Domingo P, Mocroft A, Lundgren JD, Kirk O, EuroSIDA study group: Tuberculosis among HIV-positive patients across Europe: changes over time and risk factors. AIDS 2011, 25:1505-1513.

6. Marais BJ, Rabie H, Cotton MF: TB and HIV in children - advances in prevention and management. Paediatr Respir Rev 2011, 12:39-45.

7. World Health Organization: Global HIV/AIDS response. Progress report 2011.[http://whqlibdoc.who.int/publications/2011/9789241502986_eng.pdf].

8. Judd A, Doerholt K, Tookey PA, Sharland M, Riordan A, Menson E, Novelli V, Lyall EG, Masters J, Tudor-Williams G, Duong T, Gibb DM, Collaborative HIV Paediatric Study (CHIPS), National Study of HIV in Pregnancy and Childhood (NSHPC): Morbidity, mortality, and response to treatment by children in the United Kingdom and Ireland with perinatally acquired HIV infection during 1996-2006: planning for teenage and adult care. Clin Infect Dis 2007, 45:918-924.

9. de Martino M, Tovo PA, Balducci M, Galli L, Gabiano C, Rezza G, Pezzotti P. Reduction in mortality with availability of antiretroviral therapy for children with perinatal HIV-1 infection. Italian Register for HIV Infection in Children and the Italian National AIDS Registry. JAMA 2000, 284:190-197.

10. Patel K, Hernán MA, Williams PL, Seeger JD, Mclntosh K, Van Dyke RB, Seage GR 3rd, Pediatric AIDS Clinical Trials Group 219/219C Study Team: Long-term effectiveness of highly active antiretroviral therapy on the survival of children and adolescents with HIV infection: a 10-year followup study. Clin Infect Dis 2008, 46:507-515.

11. KIDS-ART-LINC Collaboration: Low risk of death, but substantial program attrition in pediatric HIV treatment cohorts in Sub-Saharan Africa. $J$ Acquir Immune Defic Syndr 2008, 49:523-531.

12. Lumbiganon $P$, Kariminia A, Aurpibul L, Hansudewechakul R, Puthanakit T, Kurniati N, Kumarasamy N, Chokephaibulkit K, Nik Yusoff NK, Vonthanak S, Moy FS, Razali KA, Nallusamy R, Sohn AH, TREAT Asia Pediatric HIV Observational Database (TApHOD): Survival of HIV-infected children: a cohort study from the Asia-Pacific region. J Acquir Immune Defic Syndr 2011, 56:365-371.

13. Global update on HIV treatment 2013: results, impacts and opportunities. [http://apps.who.int/iris/bitstream/10665/85326/1/ 9789241505734_eng.pdf].

14. Luo C, Chintu C, Bhat G, Raviglione M, Diwan V, DuPont HL, Zumla A: Human immunodeficiency virus type-1 infection in Zambian children with tuberculosis: changing seroprevalence and evaluation of a thioacetazone-free regimen. Tuber Lung Dis 1994, 75:110-115.

15. Fairlie L, Beylis NC, Reubenson G, Moore DP, Madhi SA: High prevalence of childhood multi-drug resistant tuberculosis in Johannesburg, South Africa: a cross sectional study. BMC Infect Dis 2011, 11:28.

16. Karande S, Bhalke S, Kelkar A, Ahuja S, Kulkarni M, Mathur M: Utility of clinically-directed selective screening to diagnose HIV infection in hospitalized children in Bombay, India. J Trop Pediatr 2002, 48:149-155.

17. Matos TP, Kritski AL, Ruffino Netto A: Epidemiological aspects of tuberculosis in children and adolescents in Rio de Janeiro. J Pediatr (Rio J) 2012, 88:335-340.

18. Moyo S, Verver $S$, Mahomed $H$, Hawkridge A, Kibel M, Hatherill M Tameris M, Geldenhuys H, Hanekom W, Hussey G: Age-related tuberculosis incidence and severity in children under 5 years of age in Cape Town, South Africa. Int J Tuberc Lung Dis 2010, 14:149-154.

19. Espinal MA, Reingold AL, Pérez G, Camilo E, Soto S, Cruz E, Matos N, Gonzalez G: Human immunodeficiency virus infection in children with tuberculosis in Santo Domingo, Dominican Republic: prevalence, clinical findings, and response to antituberculosis treatment. J Acquir Immune Defic Syndr Hum Retrovirol 1996, 13:155-159. 
20. Jeena PM, Pillay P, Pillay T, Coovadia HM: Impact of HIV-1 co-infection on presentation and hospital-related mortality in children with culture proven pulmonary tuberculosis in Durban, South Africa. Int J Tuberc Lung Dis 2002, 6:672-678.

21. Mukadi YD, Wiktor SZ, Coulibaly IM, Coulibaly D, Mbengue A, Folquet AM, Ackah A, Sassan-Morokro M, Bonnard D, Maurice C, Nolan C, Kreiss JK, Greenberg AE: Impact of HIV infection on the development, clinical presentation, and outcome of tuberculosis among children in Abidjan, Côte d'Ivoire. AIDS 1997, 11:1151-1158.

22. Cohen JM, Whittaker E, Walters S, Lyall H, Tudor-Williams G, Kampmann B: Presentation, diagnosis and management of tuberculosis in HIV-infected children in the UK. HIV Med 2008, 9:277-284.

23. Thomas P, Bornschlegel K, Singh TP, Abrams EJ, Cervia J, Fikrig S, Lambert G, Mendez H, Kaye K, Bertolli J: Tuberculosis in human immunodeficiency virus-infected and human immunodeficiency virusexposed children in New York City. The New York City Pediatric Spectrum of HIV Disease Consortium. Pediatr Infect Dis J 2000, 19:700-706.

24. Walters E, Cotton MF, Rabie H, Schaaf HS, Walters LO, Marais BJ: Clinical presentation and outcome of tuberculosis in human immunodeficiency virus infected children on anti-retroviral therapy. BMC Pediatr 2008, 8:1.

25. Jensen J, Álvaro-Meca A, Micheloud D, Díaz A, Resino S: Reduction in mycobacterial disease among HIV-infected children in the highly active antiretroviral therapy era (1997-2008). Pediatr Infect Dis J 2012, 31:278-283.

26. Sonnenberg P, Glynn JR, Fielding K, Murray J, Godfrey-Faussett P, Shearer S: How soon after infection with HIV does the risk of tuberculosis start to increase? A retrospective cohort study in South African gold miners. J Infect Dis 2005, 191:150-158.

27. Geldmacher C, Zumla A, Hoelscher M: Interaction between HIV and Mycobacterium tuberculosis: HIV-1-induced CD4 T-cell depletion and the development of active tuberculosis. Curr Opin HIV AIDS 2012, 7:268-275.

28. Naing C, Mak JW, Maung M, Wong SF, Kassim Al: Meta-analysis: the association between HIV infection and extrapulmonary tuberculosis. Lung 2013, 191:27-34.

29. Patel NR, Swan K, Li X, Tachado SD, Koziel H: Impaired M. tuberculosismediated apoptosis in alveolar macrophages from HIV+ persons: potential role of IL-10 and BCL-3. J Leukoc Biol 2009, 86:53-60.

30. Pontillo A, Carvalho MS, Kamada AJ, Moura R, Schindler HC, Duarte AJ, Crovella S: Susceptibility to Mycobacterium tuberculosis infection in HIVpositive patients is associated with CARD8 genetic variant. J Acquir Immune Defic Syndr 2013, 63:147-151.

31. WHO, UATLD: Guidance for national tuberculosis and HIV programmes on the management of tuberculosis in HIV-infected children: Recommendations for a public health approach.[http://www.theunion. org/index.php/en/resources/technical-publications/item/759-guidance-fornational-tuberculosis-and-hiv-programmes-on-the-management-oftuberculosis-in-hiv-infected-children-recommendations-for-a-public-healthapproach].

32. Leeds IL, Magee MJ, Kurbatova EV, del Rio C, Blumberg HM, Leonard MK, Kraft CS: Site of extrapulmonary tuberculosis is associated with HIV infection. Clin Infect Dis 2012, 55:75-81.

33. Soeters M, de Vries AM, Kimpen JL, Donald PR, Schaaf HS: Clinical features and outcome in children admitted to a TB hospital in the Western Cape-the influence of HIV infection and drug resistance. $S$ Afr Med J 2005, 95:602-606.

34. Chan SP, Birnbaum J, Rao M, Steiner P: Clinical manifestation and outcome of tuberculosis in children with acquired immunodeficiency syndrome. Pediatr Infect Dis J 1996, 15:443-447.

35. Hesseling AC, Westra AE, Werschkull H, Donald PR, Beyers N, Hussey GD, ElSadr W, Schaaf HS: Outcome of HIV infected children with culture confirmed tuberculosis. Arch Dis Child 2005, 90:1171-1174.

36. Zar HJ, Hanslo D, Apolles P, Swingler G, Hussey G: Induced sputum versus gastric lavage for microbiological confirmation of pulmonary tuberculosis in infants and young children: a prospective study. Lancet 2005, 365:130-134.

37. Moore HA, Apolles P, de Villiers PJ, Zar HJ: Sputum induction for microbiological diagnosis of childhood pulmonary tuberculosis in a community setting. Int J Tuberc Lung Dis 2011, 15:1185-1190.

38. European Centre for Disease Prevention and Control: World Tuberculosis Day 2011. Three key messages on childhood tuberculosis. Key message 3. Stockholm. 2011 [http://ecdc.europa.eu/en/publications/Publications/ 1103_TB_SUR_2009.pdf].
39. WHO Information note 2013. Xpert MTB/RIF increases timely TB detection among people living with HIV and saves lives. [http://Www who.int/tb/challenges/hiv/Xpert_TBHIV_Information_Note_final.pdf].

40. Nicol MP, Workman L, Isaacs W, Munro J, Black F, Eley B, Boehme CC, Zemanay W, Zar HJ: Accuracy of the Xpert MTB/RIF test for the diagnosis of pulmonary tuberculosis in children admitted to hospital in Cape Town, South Africa: a descriptive study. Lancet Infect Dis 2011, 11:819-824.

41. Bates M, O'Grady J, Maeurer M, Tembo J, Chilukutu L, Chabala C, Kasonde R, Mulota P, Mzyece J, Chomba M, Mukonda L, Mumba M, Kapata N, Rachow A, Clowes P, Hoelscher M, Mwaba P, Zumla A: Assessment of the Xpert MTB/RIF assay for diagnosis of tuberculosis with gastric lavage aspirates in children in sub-Saharan Africa: a prospective descriptive study. Lancet Infect Dis 2013, 13:36-42.

42. Walters E, Gie RP, Hesseling AC, Friedrich SO, Diacon AH, Gie RP: Rapid diagnosis of pediatric intrathoracic tuberculosis from stool samples using the Xpert MTB/RIF Assay: a pilot study. Pediatr Infect Dis J 2012, 31:1316.

43. Nicol MP, Spiers K, Workman L, Isaacs W, Munro J, Black F, Zemanay W, Zar HJ: Xpert MTB/RIF testing of stool samples for the diagnosis of pulmonary tuberculosis in children. Clin Infect Dis 2013, 57:18-21.

44. Zar HJ, Workman L, Isaacs W, Munro J, Black F, Eley B, Allen V, Boehme CC, Zemanay W, Nicol MP: Rapid molecular diagnosis of pulmonary tuberculosis in children using nasopharyngeal specimens. Clin Infect Dis 2012, 55:1088-1095.

45. Lawn SD, Kerkhoff AD, Vogt M, Wood R: Diagnostic accuracy of a lowcost, urine antigen, point-of-care screening assay for HIV-associated pulmonary tuberculosis before antiretroviral therapy: a descriptive study. Lancet Infect Dis 2012, 12:201-209.

46. Denkinger $C M$, Dheda K, Pai M: Guidelines on interferon- $\gamma$ release assays for tuberculosis infection: concordance, discordance or confusion? Clin Microbiol Infect 2011, 17:806-814.

47. Mandalakas AM, Detjen AK, Hesseling AC: Can we accurately diagnose tuberculosis infection in children? Pediatr Infect Dis J 2011, 30:817-818.

48. Mandalakas AM, van Wyk S, Kirchner HL, Walzl G, Cotton M, Rabie H, Kriel B, Gie RP, Schaaf HS, Hesseling AC: Detecting tuberculosis infection in HIVinfected children: a study of diagnostic accuracy, confounding and interaction. Pediatr Infect Dis J 2013, 32:111-118.

49. Liebeschuetz S, Bamber S, Ewer K, Deeks J, Pathan AA, Lalvani A: Diagnosis of tuberculosis in South African children with a T-cell-based assay: a prospective cohort study. Lancet 2004, 364:2196-2203.

50. Davies MA, Connell T, Johannisen C, Wood K, Pienaar S, Wilkinson KA, Wilkinson RJ, Zar HJ, Eley B, Beatty D, Curtis N, Nicol MP: Detection of tuberculosis in HIV-infected children using an enzyme-linked immunospot assay. AIDS 2009, 23:961-969.

51. Ramírez-Cardich ME, Kawai V, Oberhelman RA, Bautista CT, Castillo ME, Gilman RH: Clinical correlates of tuberculosis co-infection in HIV-infected children hospitalized in Peru. Int J Infect Dis 2006, 10:278-281.

52. Palme IB, Gudetta B, Bruchfeld J, Muhe L, Giesecke J: Impact of human immunodeficiency virus 1 infection on clinical presentation, treatment outcome and survival in a cohort of Ethiopian children with tuberculosis. Pediatr Infect Dis J 2002, 21:1053-1061.

53. Whittaker E, Zar HJ: Promising directions in the diagnosis of childhood tuberculosis. Expert Rev Respir Med 2012, 6:385-395.

54. Chintu C, Mudenda V, Lucas S, Nunn A, Lishimpi K, Maswahu D, Kasolo F, Mwaba P, Bhat G, Terunuma H, Zumla A, UNZA-UCLMS Project Paediatric Post-mortem Study Group: Lung diseases at necropsy in African children dying from respiratory illnesses: a descriptive necropsy study. Lancet 2002, 360:985-990.

55. Edmonds A, Lusiama J, Napravnik S, Kitetele F, Van Rie A, Behets F: Antiretroviral therapy reduces incident tuberculosis in HIV-infected children. Int J Epidemiol 2009, 38:1612-1621.

56. Abuogi LL, Mwachari C, Leslie HH, Shade SB, Otieno J, Yienya N, Sanguli L, Amukoye $E$, Cohen CR: Impact of expanded antiretroviral use on incidence and prevalence of tuberculosis in children with HIV in Kenya. Int J Tuberc Lung Dis 2013, 17:1291-1297.

57. Martinson NA, Moultrie H, van Niekerk R, Barry G, Coovadia A, Cotton M, Violari A, Gray GE, Chaisson RE, McIntyre JA, Meyers T: HAART and risk of tuberculosis in HIV-infected South African children: a multi-site retrospective cohort. Int J Tuberc Lung Dis 2009, 13:862-867.

58. Dangor Z, IzU A, Hillier K, Solomon F, Beylis N, Moore DP, Nunes MC, Madhi SA: Impact of the Antiretroviral Treatment Program on the 
Burden of Hospitalization for Culture-confirmed Tuberculosis in South African Children: A Time-series Analysis. Pediatr Infect Dis J 2013, 32:972-977.

59. World Health Organisation: Consolidated guidelines on the use of antiretroviral drugs for treating and preventing HIV infection: recommendations for a public health approach. 2013 [http://apps.who. int/iris/bitstream/10665/85321/1/9789241505727_eng.pdf].

60. Abdool Karim SS, Naidoo K, Grobler A, Padayatchi N, Baxter C, Gray AL, Gengiah T, Gengiah S, Naidoo A, Jithoo N, Nair G, El-Sadr WM, Friedland G, Abdool Karim Q: Integration of antiretroviral therapy with tuberculosis treatment. N Engl J Med 2011, 365:1492-1501.

61. Blanc FX, Sok T, Laureillard D, Borand L, Rekacewicz C, Nerrienet E, Madec Y, Marcy O, Chan S, Prak N, Kim C, Lak KK, Hak C, Dim B, Sin Cl, Sun S, Guillard B, Sar B, Vong S, Fernandez M, Fox L, Delfraissy JF, Goldfeld AE, CAMELIA (ANRS 1295-CIPRA KH001) Study Team: Earlier versus later start of antiretroviral therapy in HIV-infected adults with tuberculosis. N Engl $\int$ Med 2011, 365:1471-1481.

62. Havlir DV, Kendall MA, Ive P, Kumwenda J, Swindells S, Qasba SS, Luetkemeyer AF, Hogg E, Rooney JF, Wu X, Hosseinipour MC, Lalloo U, Veloso VG, Some FF, Kumarasamy N, Padayatchi N, Santos BR, Reid S, Hakim J, Mohapi L, Mugyenyi P, Sanchez J, Lama JR, Pape JW, Sanchez A, Asmelash A, Moko E, Sawe F, Andersen J, Sanne I, AIDS Clinical Trials Group Study A5221: Timing of antiretroviral therapy for HIV-1 infection and tuberculosis. N Engl J Med 2011, 365:1482-1491.

63. Yotebieng M, Van Rie A, Moultrie H, Cole SR, Adimora A, Behets F, Meyers T: Effect on mortality and virological response of delaying antiretroviral therapy initiation in children receiving tuberculosis treatment. AIDS 2010, 24:1341-1349.

64. Food and Drug Administration (U.S.): Sustiva (efavirenz) pediatric patients labeling update. 2013 [http://www.fda.gov/ForConsumers/ByAudience/ ForPatientAdvocates/HIVandAIDSActivities/ucm350744.htm]

65. Ren Y, Nuttall JJ, Egbers C, Eley BS, Meyers TM, Smith PJ, Maartens G, Mclleron HM: Effect of rifampicin on lopinavir pharmacokinetics in HIVinfected children with tuberculosis. J Acquir Immune Defic Syndr 2008, 47:566-569.

66. Frohoff C, Moodley M, Fairlie L, Coovadia A, Moultrie H, Kuhn L, Meyers T: Antiretroviral therapy outcomes in HIV-infected children after adjusting protease inhibitor dosing during tuberculosis treatment. PLoS One 2011, 6:17273.

67. Shah I, Swaminathan S, Ramachandran G, Kumar AK, Goray A, Chaddha U, Tayal S, Lala M: Serum Nevirapine and Efavirenz concentrations and effect of concomitant rifampicin in HIV infected children on antiretroviral therapy. Indian Pediatr 2011, 48:943-947.

68. ARROW Trial team, Kekitiinwa A, Cook A, Nathoo K, Mugyenyi P, NahiryaNtege P, Bakeera-Kitaka S, Thomason M, Bwakura-Dangarembizi M, Musiime V, Munderi P, Naidoo-James B, Vhembo T, Tumusiime C, Katuramu R, Crawley J, Prendergast AJ, Musoke P, Walker AS, Gibb DM: Routine versus clinically driven laboratory monitoring and first-line antiretroviral therapy strategies in African children with HIV (ARROW): a 5-year open-label randomised factorial trial. Lancet 2013, 381:1391-1403.

69. Andrews JR, Shah NS, Gandhi N, Moll T, Friedland G, Tugela Ferry Care and Research (TF CARES) Collaboration: Multidrug-resistant and extensively drug-resistant tuberculosis: implications for the HIV epidemic and antiretroviral therapy rollout in South Africa. J Infect Dis 2007, 196(Suppl 3):482-490.

70. Satti H, McLaughlin MM, Omotayo DB, Keshavjee S, Becerra MC Mukherjee JS, Seung KJ: Outcomes of comprehensive care for children empirically treated for multidrug-resistant tuberculosis in a setting of high HIV prevalence. PLoS One 2012, 7:37114.

71. Thomas TA, Shenoi SV, Heysell SK, Eksteen FJ, Sunkari VB, Gandhi NR, Friedland G, Shah NS: Extensively drug-resistant tuberculosis in children with human immunodeficiency virus in rural South Africa. Int $J$ Tuberc Lung Dis 2010, 14:1244-1251.

72. Seddon JA, Hesseling AC, Willemse M, Donald PR, Schaaf HS: Cultureconfirmed multidrug-resistant tuberculosis in children: clinical features, treatment, and outcome. Clin Infect Dis 2012, 54:157-166.

73. Schaaf HS, Marais BJ: Management of multidrug-resistant tuberculosis in children: a survival guide for paediatricians. Paediatr Respir Rev 2011, 12:31-38.

74. Poorana Ganga Devi NP, Swaminathan S: Drug-resistant tuberculosis: pediatric guidelines. Curr Infect Dis Rep 2013, 15:356-363.
75. USAID: The Sentinel Project for Pediatric Drug-Resistant Tuberculosis. Management of drug-resistant tuberculosis in children: a field guide. 2012 [http://sentinelproject.files.wordpress.com/2012/11/ sentinel_project_field_guide_2012.pdf].

76. Seddon JA, Hesseling AC, Godfrey-Faussett P, Schaaf HS: High treatment success in children treated for multidrug-resistant tuberculosis: an observational cohort study. Thorax 2013, Epub ahead of print.

77. Laureillard D, Marcy O, Madec Y, Chea S, Chan S, Borand L, Fernandez M, Prak N, Kim C, Dim B, Nerrienet E, Sok T, Delfraissy JF, Goldfeld AE, Blanc FX CAMELIA (ANRS 1295 - CIPRA KH001) Study Team: Paradoxical tuberculosis-associated immune reconstitution inflammatory syndrome after early initiation of antiretroviral therapy in a randomized clinical trial. AIDS 2013, 27:2577-2586.

78. Leroy V, Msellati P, Lepage P, Batungwanayo J, Hitimana DG, Taelman H, Bogaerts J, Boineau F, Van de Perre $P$, Simonon A: Four years of natural history of HIV-1 infection in african women: a prospective cohort study in Kigali (Rwanda), 1988-1993. J Acquir Immune Defic Syndr Hum Retrovirol 1995, 9:415-421.

79. Donald PR, Marais BJ, Barry CE 3rd: Age and the epidemiology and pathogenesis of tuberculosis. Lancet 2010, 375:1852-1854.

80. Bruchfeld J, Aderaye G, Palme IB, Bjorvatn B, Britton S, Feleke Y, Källenius G, Lindquist $L$ : Evaluation of outpatients with suspected pulmonary tuberculosis in a high HIV prevalence setting in Ethiopia: clinical, diagnostic and epidemiological characteristics. Scand J Infect Dis 2002, 34:331-337.

81. Pillay T, Sturm AW, Khan M, Adhikari M, Moodley J, Connolly C, Moodley D, Padayatchi N, Ramjee A, Coovadia HM, Sullivan JL: Vertical transmission of Mycobacterium tuberculosis in KwaZulu Natal: impact of HIV-1 coinfection. Int J Tuberc Lung Dis 2004, 8:59-69.

82. Gupta A, Bhosale R, Kinikar A, Gupte N, Bharadwaj R, Kagal A, Joshi S, Khandekar M, Karmarkar A, Kulkarni V, Sastry J, Mave V, Suryavanshi N, Thakar M, Kulkarni S, Tripathy S, Sambarey P, Patil S, Paranjape R, Bollinger RC, Jamkar A, Six Week Extended-Dose Nevirapine (SWEN) India Study Team: Maternal tuberculosis: a risk factor for mother-to-child transmission of human immunodeficiency virus. J Infect Dis 2011, 203:358-363.

83. Adhikari M, Jeena P, Bobat R, Archary M, Naidoo K, Coutsoudis A, Singh R, Nair N: HIV-Associated Tuberculosis in the Newborn and Young Infant. Int J Pediatr 2011, 2011:354208.

84. Zar HJ, Cotton MF, Strauss S, Karpakis J, Hussey G, Schaaf HS, Rabie H, Lombard CJ: Effect of isoniazid prophylaxis on mortality and incidence of tuberculosis in children with HIV: randomised controlled trial. BMJ 2007, 334:136.

85. Madhi SA, Nachman S, Violari A, Kim S, Cotton MF, Bobat R, Jean-Philippe P, McSherry G, Mitchell C, P1041 Study Team: Primary isoniazid prophylaxis against tuberculosis in HIV-exposed children. N Engl J Med 2011, 365:21-31.

86. Report of the HIV/TB Research meeting held in conjunction with the 20th Conference on Retroviruses and Opportunistic Infections (CROI 2013). [http://www.who.int/tb/challenges/hiv/tbhiv_at_croi_2013_report. pdf].

87. The PREVENT TB Study. Rifapentine + isoniazid to prevent TB among people living with HIV: interim data and pending issues. [http://www. who.int/tb/challenges/hiv/ sterling_prevent_tb_study_26_hiv_croi_03mar2013.pdf].

doi:10.1186/1471-2334-14-S1-S5

Cite this article as: Venturini et al:: Tuberculosis and HIV co-infection in children. BMC Infectious Diseases 2014 14(Suppl 1):S5. 